\title{
Editorial
}

\section{Supersymmetry beyond the NMSSM}

\author{
Florian Staub, ${ }^{1}$ Mark D. Goodsell, ${ }^{2,3}$ Michal Malinský, ${ }^{4}$ and Kai Schmidt-Hoberg ${ }^{5}$ \\ ${ }^{1}$ Theory Division, CERN, 1211 Geneva 23, Switzerland \\ ${ }^{2}$ Sorbonne Universités, UPMC Univ. Paris 06, UMR 7589, LPTHE, 75005 Paris, France \\ ${ }^{3}$ CNRS, UMR 7589, LPTHE, 75005 Paris, France \\ ${ }^{4}$ Institute of Particle and Nuclear Physics, Faculty of Mathematics and Physics, Charles University in Prague, \\ $V$ Holešovičkách 2, 18000 Praha 8, Czech Republic \\ ${ }^{5}$ Deutsches Elektronen-Synchrotron (DESY), Notkestraße 85, 22607 Hamburg, Germany
}

Correspondence should be addressed to Florian Staub; florian.staub@cern.ch

Received 13 August 2015; Accepted 16 August 2015

Copyright (C) 2015 Florian Staub et al. This is an open access article distributed under the Creative Commons Attribution License, which permits unrestricted use, distribution, and reproduction in any medium, provided the original work is properly cited. The publication of this article was funded by SCOAP $^{3}$.

With the start of run II of the Large Hadron Collider (LHC) a new milestone of high energy particle physics has been reached. Run I was on the one side a great success because of the discovery of a fundamental scalar with all expected properties of the so long-expected standard model (SM) Higgs boson $[1,2]$. On the other side, no clear sign of any physics beyond the SM has shown up. This has placed severe constraints on the simplest scenarios for new physics. In particular the minimal realization of supersymmetry (SUSY), the minimal supersymmetric standard model (MSSM), has lost some of its appeal since it is no longer clear that it can address the question of naturalness. In general, there are two possibilities to explain the measured mass of $125 \mathrm{GeV}$ within the MSSM: either heavy SUSY masses are needed, or the stop mixing has to be very large. In the first case, a new hierarchy problem is introduced, while the second can be dangerous because of charge and colour breaking minima [3-7].

These observations together with the other null results from SUSY searches have created a much stronger interest in nonminimal SUSY models in recent years. Extensions of the MSSM not only might provide a natural explanation for the size of the Higgs mass, but also can explain other (non)observations such as neutrino masses (e.g., in seesaw models) and the missing signals for supersymmetry (e.g., due to broken R-parity). More theoretical issues of the MSSM can also be addressed, such as the $\mu$-problem in singlet extensions and the origin of R-parity in models with gauged $B-L$.
This special issue discusses several theoretical and experimental aspects of nonminimal SUSY models. To set the scene, the first article reconsiders the beginnings of supersymmetry and reveals its secret history: "Supersymmetry: Early Roots That Did Not Grow" by C. Jarlskog points out that the fundamental concepts of SUSY were already known in the 1940s.

Our first research article is inspired by top-down approaches: "Phenomenological Hints from a Class of String Motivated Model Constructions" by H. P. Nilles connects string theoretical considerations with LHC phenomenology. Generic predictions of a specific class of string motivated models are presented.

The next two articles consider singlet extensions of the MSSM. In "Two Higgs Bosons near $125 \mathrm{GeV}$ in the Complex NMSSM and the LHC Run-I Data" by S. Moretti and S. Munir the properties of the Higgs fields in the $Z_{3}$ invariant singlet extensions with complex parameters are discussed. In particular, they consider the case where two Higgs bosons have such close masses that they are not separately resolved by the LHC experiments and find that for some parameter points this can provide a better fit to the data than a single Higgs.

The authors M. Ratz and P. K. S. Vaudrevange, in "Singlet Extensions of the MSSM with $Z_{4}^{R}$ Symmetry," consider a different discrete symmetry which allows for more parameters in the superpotential. It is shown that the potentially 
dangerous linear term can be avoided in this class of models.

As mentioned, one of the best possibilities to hide SUSY at the LHC is to assume that R-parity is not conserved. In this case the limits on SUSY masses are significantly reduced. The two articles "Status of LHC searches for SUSY without Rparity" by R. Franceschini and "Searches for Prompt R-ParityViolating Supersymmetry at the LHC" by A. Redelbach discuss the current status of SUSY searches at the LHC in the context of R-parity violation. Together these provide an excellent and up-to-date reference for this class of searches.

SUSY models which include an explanation for the neutrino masses and mixing angles also predict nonvanishing rates for lepton-flavour violating (LFV) observables like $\mu \rightarrow$ e $\gamma$ or $\mu \rightarrow 3 e$. The article "Lepton Flavor Violation beyond the MSSM" by A. Vicente discusses the constraints on different SUSY models derived by the strong experimental bounds for LFV observables.

A possible origin of R-parity in SUSY models is the presence of an Abelian $B-L$ gauge group which is broken in a specific way to retain an unbroken $Z_{2}$ subgroup. These models are always accompanied by an extended Higgs sector. The article "The Higgs Sector of the Minimal SUSY $B-L$ Model" by L. Basso discusses the Higgs properties in the minimal SUSY model with a gauged $U(1)_{B-L}$ and unbroken R-parity.

Dirac gaugino models have recently attracted much attention in the literature due to their many attractive properties over their Majorana counterparts (as in the MSSM). However when in addition an unbroken R-symmetry is imposed, such models are expected to have difficulties to explain the observed Higgs mass: since the left- and right-handed stops cannot mix, the stop radiative corrections to the Higgs are suppressed. The article "Two-Loop Correction to the Higgs Boson Mass in the MRSSM" by P. Diessner et al. discusses the minimal R-symmetric SUSY model and shows that the observed Higgs mass can be generated via large radiative corrections involving other states. It includes all of the latest corrections which have recently become available, equivalent to the precision available for the MSSM: an illustration of the rapid recent developments in beyond-the-MSSM phenomenology.

The above articles give a cross section of current thinking in this rapidly expanding area. We hope that this special issue will therefore prove to be a useful resource for those entering the field and experts alike, as we eagerly wait for the data with, we hope, the first signs of what may lie beyond the (minimal supersymmetric) standard model.

\section{Acknowledgment}

We sincerely thank all authors for their contribution to this special issue.

\section{References}

[1] S. Chatrchyan, V. Khachatryan, A. M. Sirunyan et al., "Observation of a new boson at a mass of $125 \mathrm{GeV}$ with the CMS experiment at the LHC," Physics Letters B, vol. 716, no. 1, pp. 30-61, 2012.

[2] G. Aad, T. Abajya, B. Abbott et al., "Observation of a new particle in the search for the standard model Higgs boson with the ATLAS detector at the LHC," Physics Letters B, vol. 716, no. 1, pp. 1-29, 2012.

[3] J. E. Camargo-Molina, B. O'Leary, W. Porod, and F. Staub, "Stability of the CMSSM against sfermion VEVs," Journal of High Energy Physics, vol. 2013, no. 12, article 103, 2013.

[4] N. Blinov and D. E. Morrissey, "Vacuum stability and the MSSM Higgs mass," Journal of High Energy Physics, vol. 2014, no. 3, article 106, 2014.

[5] D. Chowdhury, R. M. Godbole, K. A. Mohan, and S. K. Vempati, "Charge and color breaking constraints in MSSM after the Higgs discovery at LHC," Journal of High Energy Physics, vol. 2014, article 110, 2014.

[6] J. E. Camargo-Molina, B. Garbrecht, B. O'Leary, W. Porod, and F. Staub, "Constraining the Natural MSSM through tunneling to color-breaking vacua at zero and non-zero temperature," Physics Letters B, vol. 737, pp. 156-161, 2014.

[7] U. Chattopadhyay and A. Dey, "Exploring MSSM for charge and color breaking and other constraints in the context of Higgs@125 GeV,' Journal of High Energy Physics, vol. 2014, no. 11, article 161, 2014.
Florian Staub

Mark D. Goodsell

Michal Malinský

Kai Schmidt-Hoberg 

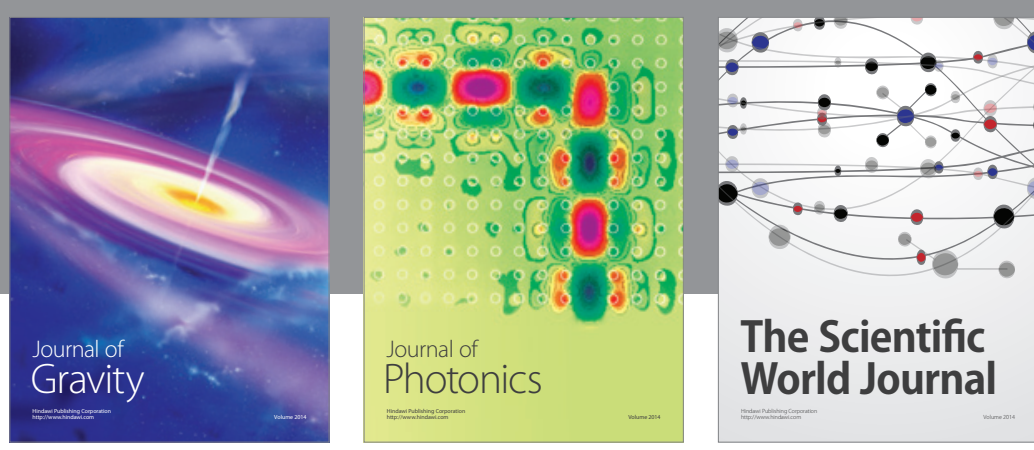

The Scientific World Journal
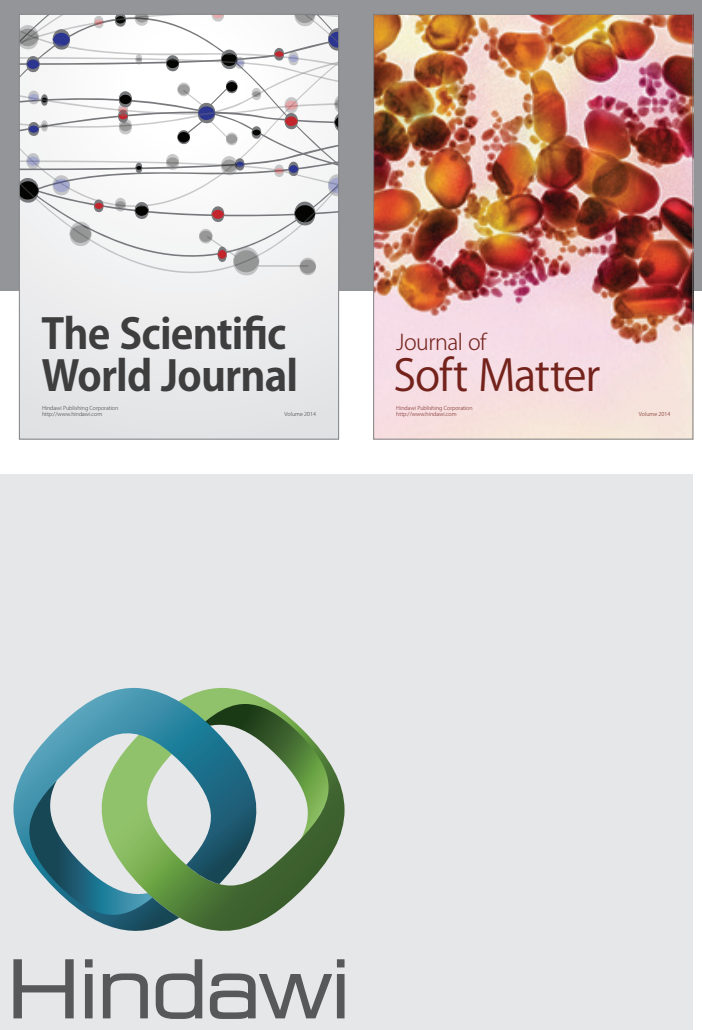

Submit your manuscripts at

http://www.hindawi.com

nternational Journal of

Statistical Mechanics
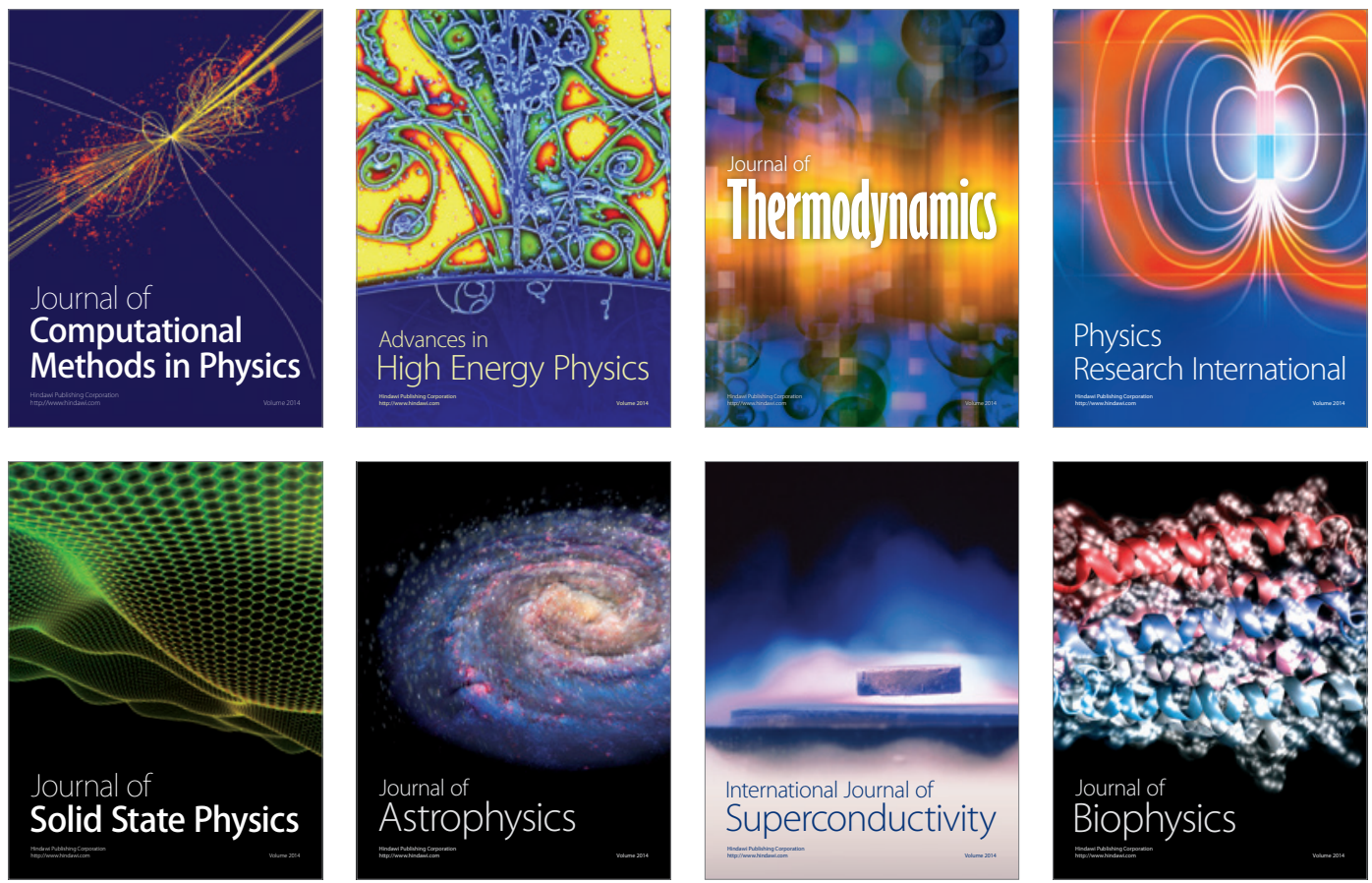
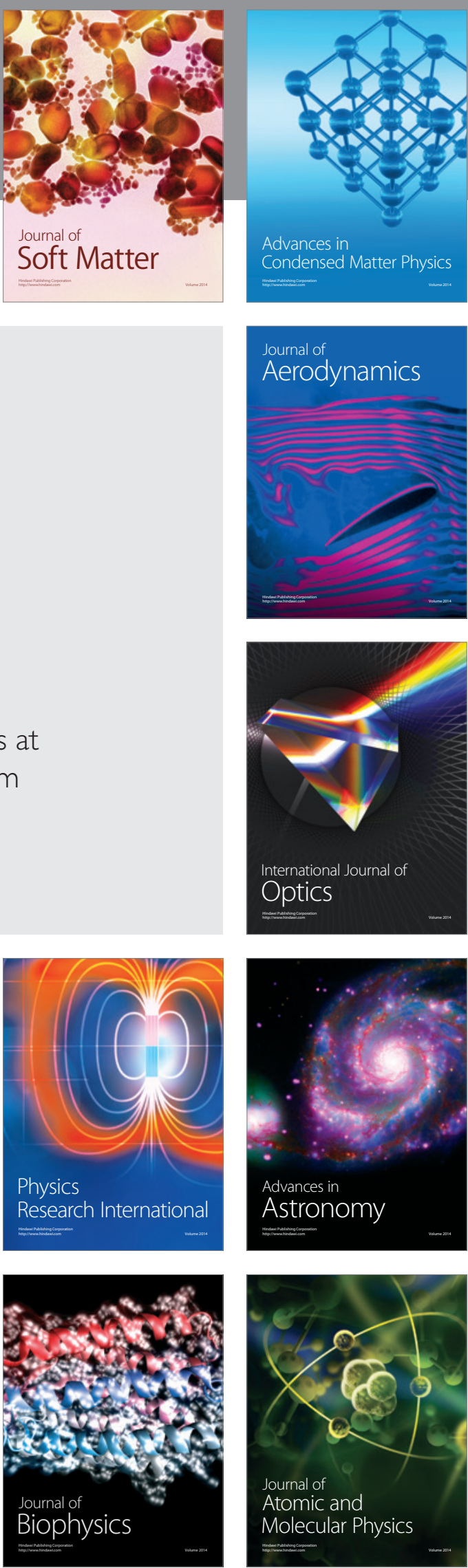